\title{
Fibromixoma acral superficial: Revisão da Literatura*
}

\section{Superficial Acral Fibromyxoma: Literature Review}

\author{
Bruno Eiras Crepaldi ${ }^{1}$ Ruan Dalbem Soares ${ }^{1}{ }^{10}$ Fábio Duque Silveira ${ }^{1}$ Raul Itocazo Taira ${ }^{1}$ \\ Celso Kiyoshi Hirakawa ${ }^{1}$ Marcelo Hide Matsumoto ${ }^{1}$ \\ ${ }^{1}$ Grupo de Cirurgia da Mão e Microcirurgia, Hospital Santa Marcelina \\ de Itaquera, São Paulo, SP, Brasil \\ Endereço para correspondência Ruan Dalbem Soares, MD, Grupo de \\ Cirurgia da Mão e Microcirurgia, Hospital Santa Marcelina de Itaquera, \\ Rua Gonçalo da Cunha, 43, Apto 56, Chácara Inglesa, \\ Rev Bras Ortop 2019;54:491-496. \\ São Paulo, 04140-040, SP, Brasil (e-mail: ruansoares1@hotmail.com).
}

\section{Resumo}

Palavras-chave

- neoplasias de tecidos moles

- fibroma/patologia

- fibroma/terapia

- neoplasias cutâneas
O fibromixoma acral superficial é um tumor raro de tecidos moles. Geralmente se manifesta por meio de uma massa indolor de crescimento lento que acomete principalmente adultos do sexo masculino na quinta década de vida. Ele normalmente afeta a região distal, com aparência polipoide. A aparência histológica é de uma massa dérmica sem cápsula, com fibroblastos fusiformes em estroma mixocolagenoso. A avaliação imuno-histoquímica do fibromixoma acral superficial normalmente é positiva para CD34 e CD99, com positividade variável para o antígeno epitelial de membrana. $\mathrm{O}$ tratamento consiste na exérese completa da massa tumoral.

Foi feita uma revisão da literatura atual sobre o fibromixoma acral superficial com ênfase na quantidade de casos relatados, na localização, nos métodos diagnósticos, nas características histológicas, nos diagnósticos diferenciais, e no tratamento.

Foram encontrados na literatura atual 314 casos descritos de fibromixoma acral superficial com localização variada, principalmente em pododáctilos $(45,8 \%)$ e quirodáctilos (39,1\%). Este tumor tem acometimento ligeiramente superior em homens (61\%), e enorme variabilidade na faixa etária de acometimento.

O fibromixoma acral superficial é um tumor de tecido mole único que deve entrar no diagnóstico diferencial das lesões periungueais e subungueais acrais; o tratamento consiste da exérese simples. Mais estudos são necessários para que se conheça melhor essa patologia, descrita em 2001.

Superficial acral fibromyxoma is a benign and rare tumor of the soft tissues. It is a benign slow-growing soft tissue lesion occurring in males in the fifth decade. Clinically, superficial acral fibromyxoma generally presents on the distal aspect of the hands or feet, with a polypoid or dome shaped appearance. Histologically, it appears as a nonencapsulated soft-tissue tumor of the dermis, with spindle-shaped fibroblasts in a storiform or fasciculated pattern in the myxocollagenous stroma. On the immunohistochemistry, superficial acral fibromyxoma is commonly positive for CD34 and CD99, with variable positivity for epithelial membrane antigen. The management of superficial acral fibromyxoma consists of complete surgical excision.

Trabalho desenvolvido no Grupo de Cirurgia da Mão e Microcirurgia, Hospital Santa Marcelina de Itaquera, São Paulo, SP, Brasil. Publicado Originalmente por Elsevier Editora Ltda.

recebido

13 de Agosto de 2017

aceito

31 de Outubro de 2017
DOI https://doi.org/

10.1016/j.rbo.2017.10.011. ISSN 0102-3616.
Copyright (c) 2019 by Sociedade Brasileira License terms de Ortopedia e Traumatologia. Published by Thieme Revinter Publicações Ltda, Rio de Janeiro, Brazil 


\section{Keywords}

- soft-tissue neoplasms

- fibroma/pathology

- fibroma/therapy

- skin neoplasms
A review of the current literature on superficial acral fibromyxoma was performed, with an emphasis on the number of reported cases, location, diagnostic methods, histological characteristics, differential diagnoses, and treatment.

A total of 314 reported cases of superficial acral fibromyxoma were found in the current literature, mainly in the toes (45.8\%) and fingers (39.1\%). It has a slightly greater incidence in men (61\%), and has enormous variability in the age group of occurrence. Superficial acral fibromyxoma is a single soft-tissue tumor that must enter into the differential diagnosis of periungual and subcutaneous acral lesions; its treatment consists of simple excision. More studies are needed in order to better understand this pathology, which was first described in 2001.

\section{Introdução}

Fibromixoma acral superficial (FAS) é um tumor mixoide descrito em 2001 por Fetsch et al, ${ }^{1}$ que acomete preferencialmente a região subungueal ou periungueal. Geralmente se manifesta por meio de massa indolor, com crescimento lento, $\mathrm{e}$ que acomete principalmente adultos do sexo masculino na quinta década de vida. Localiza-se normalmente na região acral das mãos e dos pés; porém, pode afetar a região do calcanhar e do tornozelo. ${ }^{2-7}$ Histórico de trauma associado é raro, e a maioria dos tumores cresce de forma assintomática. No que diz respeito à análise histológica, apresenta-se como uma neoplasia de delineação difícil e composta de células fusiformes ou estreladas, com grau variado de pleomorfismo. Apresenta células dispostas em estroma mixoide e colagenoso, com imunorreatividade para CD34, CD99, e vimentina, e focalmente para o antígeno de membrana epitelial (AME). Vasos sanguíneos predominam na área mixoide, e mastócitos costumam estar distribuídos em toda a lesão. $\mathrm{O}$ tratamento consiste tipicamente na exérese da massa completa com o intuito de evitar recidivas ou malignização, embora o tumor não apresente comportamento agressivo, apesar da presença de atipias celulares em alguns casos. Até o presente momento, não foi relatada a ocorrência de malignização na literatura. 0 seguimento pós-operatório deve ser feito devido à taxa de recorrência de 10 a $24 \%{ }^{6-8}$ Este trabalho tem como objetivo fazer uma revisão da literatura atual sobre o FAS, com ênfase na quantidade de casos relatados, na distribuição de gênero, na localização, nas características da lesão, nos métodos diagnósticos, nas características histológicas, nos diagnósticos diferenciais, e no tratamento.

\section{Casuística e Métodos}

O presente estudo de revisão da literatura sobre a tumoração descrita por Fetsch et al ${ }^{1}$ como fibromixoma acral superficial tem como ênfase estudar a quantidade de casos relatados, sua localização, as principais características histológicas, seus diagnósticos diferenciais, e o tratamento. Foi feita uma pesquisa bibliográfica no site de periódicos Pubmed, no qual foram encontrados 58 trabalhos por meio da pesquisa pelos descritores: "superficial AND acral AND fibromyxoma". Para a inserção no estudo em questão, foram estabelecidos os seguintes critérios de inclusão e exclusão:

\section{Critérios de Inclusão}

- Trabalhos escritos em língua portuguesa e/ou inglesa.

- Trabalhos completos.

- Artigos que apresentavam técnicas diagnósticas, diagnósticos diferenciais, técnicas cirúrgicas, ou relatos de caso sobre a patologia em questão.

\section{Critérios de Exclusão}

- Artigos sem resumos.

- Trabalhos escritos em línguas diferentes das mencionadas nos critérios de inclusão.

- Trabalhos cujo tema diferia do apresentado na pesquisa.

Foram excluídos desta revisão seis artigos por não terem resumos, quatro por não terem sido escritos em português ou inglês, e três por não acrescentarem informações quanto a casos relatados, localização, características histológicas, diagnósticos diferenciais e/ou tratamento da patologia.

\section{Resultados}

Foram encontrados na literatura atual 314 casos descritos de FAS com localização variada, representados na tabela 1., , $^{1,-38}$

A média da idade na qual foi feito o diagnóstico foi de 47 anos e 7 meses, mas com enorme variância; foram encontrados casos em pacientes com idades entre 4 e 91 anos.

Dos 306 casos em que a informação de gênero estava disponível, encontramos: $61 \%$ dos casos em homens, enquanto as mulheres foram responsáveis por apenas 39\% (-Fig. 1).

Com relação à localização, encontramos acometimento quase que exclusivo das mãos e dos pés, mais comumente nas regiões dos dedos. Também foi descrito acometimento em panturrilha e coxa. ${ }^{8}$

Os principais locais de acometimento ( - Fig. 2) foram os pododáctilos, responsáveis por $45,8 \%$, e, em segundo lugar, os quirodáctilos, com 39,1\% dos casos. Em 19 casos (6\%), a informação sobre a localização não estava disponível (-Fig. 2).

Tipicamente, o FAS é uma lesão de crescimento lento, solitária, geralmente indolor, com consistência entre gelatinosa e firme, e apresenta tamanho entre 0,5 e $5 \mathrm{~cm} .{ }^{1-8}$

No exame histológico, o FAS é encontrado como um tumor de derme não encapsulado, que pode se estender ao tecido subcutâneo, à fáscia subjacente ou à camada periostal. ${ }^{1}$ É 
Tabela 1 Distribuição dos casos de fibromixoma superficial acral por artigo, número de casos e localização

\begin{tabular}{|c|c|c|c|c|c|c|c|}
\hline Referências & $\begin{array}{l}\text { Casos } \\
\text { Relatados }\end{array}$ & Localização & $\begin{array}{l}\mathrm{N}^{\circ} \mathrm{de} \\
\text { casos }\end{array}$ & Referências & $\begin{array}{l}\text { Casos } \\
\text { relatados }\end{array}$ & Localização & $\begin{array}{l}\mathrm{N}^{\circ} \mathrm{de} \\
\text { casos }\end{array}$ \\
\hline $\begin{array}{l}\text { Fetsch et al }{ }^{1} \\
\text { Hum Pathol. } 2001\end{array}$ & 37 & $\begin{array}{l}\text { Pododáctilos } \\
\text { Quirodáctilos } \\
\text { Palma }\end{array}$ & $\begin{array}{l}20 \\
13 \\
4\end{array}$ & $\begin{array}{l}\text { Kazakov et al }{ }^{14} \\
\text { Dermatology. } \\
2002\end{array}$ & 2 & Pododáctilos & 2 \\
\hline $\begin{array}{l}\text { Meyerle et al }{ }^{13} \\
\text { J Am Acad } \\
\text { Dermatol. } 2004\end{array}$ & 1 & $\begin{array}{l}\text { Segundo } \\
\text { quirodáctilo } \\
\text { (subungueal) }\end{array}$ & 1 & $\begin{array}{l}\text { André et al }{ }^{10} \\
\text { Am J Dermatopathol. } 2004\end{array}$ & 1 & Hálux & 1 \\
\hline $\begin{array}{l}\text { Quaba et al }{ }^{9} \\
\text { Br J Plast Surg. } 2005\end{array}$ & 1 & Quarto quirodáctilo & 1 & $\begin{array}{l}\text { Abou-Nukta et al }{ }^{12} \\
\text { J Hand Surg Br. } 2006\end{array}$ & 1 & Unha do polegar & 1 \\
\hline $\begin{array}{l}\text { Oteo-Alvaro et al }{ }^{11} \\
\text { Arch Orthop } \\
\text { Tra Surg. } 2008\end{array}$ & 1 & Pododáctilo & 1 & $\begin{array}{l}\text { Misago et al }{ }^{17} \\
\text { J Eur Acad Derma. } \\
\text { Venereol. } 2008\end{array}$ & 1 & Ponta do hálux & 1 \\
\hline $\begin{array}{l}\text { Varikatt et al }{ }^{18} \\
\text { Skeletal Radiol. } 2008\end{array}$ & 2 & $\begin{array}{l}\text { Ponta do segundo } \\
\text { quirodáctilo }\end{array}$ & 2 & $\begin{array}{l}\text { Al-Daraji e Miettinen }^{7} \\
\text { J Cutan Pathol. } 2008\end{array}$ & 32 & $\begin{array}{l}\text { Pododáctilos } \\
\text { Quirodáctilos } \\
\text { Calcanhar }\end{array}$ & $\begin{array}{l}15 \\
13 \\
4\end{array}$ \\
\hline $\begin{array}{l}\text { Al-Daraji e Miettinen } \\
\text { Dermatol } \\
\text { Online J. } 2008\end{array}$ & 2 & $\begin{array}{l}\text { Hálux } \\
\text { Quirodáctilo }\end{array}$ & $\begin{array}{l}1 \\
1\end{array}$ & $\begin{array}{l}\text { Prescott et al } \\
\text { Br J Dermatol. } 2008\end{array}$ & 41 & $\begin{array}{l}\text { Pododáctilos } \\
\text { Quirodáctilos } \\
\text { Palma }\end{array}$ & $\begin{array}{l}29 \\
11 \\
1\end{array}$ \\
\hline $\begin{array}{l}\text { Tadío et al }{ }^{15} \\
\text { Am J Dermatophatho. } \\
2008\end{array}$ & 4 & $\begin{array}{l}\text { Hálux } \\
\text { Palma } \\
\text { Polegar } \\
\text { Quirodáctilo }\end{array}$ & $\begin{array}{l}1 \\
1 \\
1 \\
1\end{array}$ & $\begin{array}{l}\text { Luzar e Calonje }{ }^{20} \\
\text { Histopathology. } 2009\end{array}$ & 14 & $\begin{array}{l}\text { Hálux } \\
?\end{array}$ & $\begin{array}{l}8 \\
6\end{array}$ \\
\hline $\begin{array}{l}\text { Pasquinelli et al }{ }^{16} \\
\text { Ultrastruct } \\
\text { Pathol. } 2009\end{array}$ & 1 & $\begin{array}{l}\text { Segundo } \\
\text { quirodáctilo }\end{array}$ & 1 & $\begin{array}{l}\text { Wang et al }{ }^{19} \\
\text { Zhonghua Bing Li } \\
\text { Xue Za Zhi } 2009\end{array}$ & 1 & $\begin{array}{l}\text { Terceiro } \\
\text { quirodáctilo }\end{array}$ & 1 \\
\hline $\begin{array}{l}\text { Goo et } \mathrm{al}^{22} \\
\text { Ann Dermatol. } 2010\end{array}$ & 1 & $\begin{array}{l}\text { Segundo } \\
\text { quirodáctilo } \\
\text { (subungueal) }\end{array}$ & 1 & $\begin{array}{l}\text { Chattopadhyay } \\
\text { et } \mathrm{al}^{21} \\
\text { Clin Exp Dermatol. } 2010\end{array}$ & 1 & $\begin{array}{l}\text { Segundo } \\
\text { quirodáctilo } \\
\text { (subungueal) }\end{array}$ & 1 \\
\hline $\begin{array}{l}\text { Cogrel et al }{ }^{29} \\
\text { Ann Dermatol } \\
\text { Vereol. } 2010\end{array}$ & 3 & $\begin{array}{l}\text { Quirodáctilo Hálux } \\
\text { Pododáctilo }\end{array}$ & $\begin{array}{l}1 \\
1 \\
1\end{array}$ & $\begin{array}{l}\text { Fanti et al }{ }^{23} \\
\text { G Ital Dermatol } \\
\text { Venereol. } 2011\end{array}$ & 12 & $\begin{array}{l}\text { Quirodáctilos } \\
\text { Pododáctilos }\end{array}$ & $?$ \\
\hline $\begin{array}{l}\text { Messeguer et al }{ }^{4} \text { Actas } \\
\text { Dermosifiliogr. } 2012\end{array}$ & 1 & $\begin{array}{l}\text { Segundo } \\
\text { quirodáctilo }\end{array}$ & 1 & $\begin{array}{l}\text { Bem Brahim et al }{ }^{38} \\
\text { Tunis Med. } 2012\end{array}$ & 1 & Pododáctilo & 1 \\
\hline $\begin{array}{l}\text { Wakabayashi et al }{ }^{3} \\
\text { Acta Dermatovenerol } \\
\text { Croat. } 2012\end{array}$ & 1 & Hálux & 1 & $\begin{array}{l}\text { Wei e Fleegler } \\
\text { Eplasty. } 2013\end{array}$ & 1 & Polegar & 1 \\
\hline $\begin{array}{l}\text { Kwok et } \mathrm{al}^{37} \text { Clin Exp } \\
\text { Dermato. } 2013\end{array}$ & 1 & Pododáctilo & 1 & $\begin{array}{l}\text { Hwang et al }{ }^{36} \\
\text { Arch of Plast Surg. } 2013\end{array}$ & 1 & $\begin{array}{l}\text { Segundo } \\
\text { pododáctilo }\end{array}$ & 1 \\
\hline $\begin{array}{l}\text { Chabbab et al }\left.\right|^{33} \\
\text { Anales of Derma. } 2014\end{array}$ & 3 & Subungueal & 3 & $\begin{array}{l}\text { Garcia et al }{ }^{35} \\
\text { Anais } \mathrm{Br} \text { de Derma. } 2014\end{array}$ & 1 & $\begin{array}{l}\text { Quinto } \\
\text { pododáctilo }\end{array}$ & 1 \\
\hline $\begin{array}{l}\text { Park et al } 24 \\
\text { Annals of Derma. } 2014\end{array}$ & 1 & Palma & 1 & $\begin{array}{l}\text { Carranza et } \mathrm{al}^{27} \\
\text { The Am Journ of } \\
\text { Derma. } 2015\end{array}$ & 1 & Hálux & 1 \\
\hline $\begin{array}{l}\text { Schwager et al } \\
\text { Cutis. } 2015\end{array}$ & 3 & $\begin{array}{l}\text { Hálux } \\
\text { Quarto pododáctilo } \\
\text { Quinto pododáctilo }\end{array}$ & $\begin{array}{l}1 \\
1 \\
1\end{array}$ & $\begin{array}{l}\text { Raghupathi et al }{ }^{32} \\
\text { Journal of Cytol. } 2015\end{array}$ & 1 & $\begin{array}{l}\text { Hálux } \\
\text { (subungueal) }\end{array}$ & 1 \\
\hline $\begin{array}{l}\text { Grigore et al }{ }^{26} \\
\text { Clujul Med. } 2016\end{array}$ & 1 & Hálux (subungueal) & 1 & $\begin{array}{l}\text { Ramya et al }{ }^{31} \\
\text { India Jour of Derma. } 2016\end{array}$ & 1 & $\begin{array}{l}\text { Polegar } \\
\text { (subungueal) }\end{array}$ & 1 \\
\hline $\begin{array}{l}\text { Sundaramurthy et } \text { al }^{30} \\
\text { Journ Clin Diag } \\
\text { Rese. } 2016\end{array}$ & 1 & $\begin{array}{l}\text { Segundo } \\
\text { quirodáctilo }\end{array}$ & 1 & $\begin{array}{l}\text { DeFroda et al }{ }^{28} \text { Journ } \\
\text { of Ortopae. } 2016\end{array}$ & 1 & Polegar & 1 \\
\hline $\begin{array}{l}\text { Agaimy et al }{ }^{25} \\
\text { Hum Pathology. } 2016\end{array}$ & 11 & $\begin{array}{l}\text { Quirodáctilos } \\
\text { Pododáctilos } \\
\text { Calcanhar } \\
\text { Panturrilha } \\
\text { ? }\end{array}$ & $\begin{array}{l}5 \\
3 \\
1 \\
1 \\
1\end{array}$ & $\begin{array}{l}\text { Hollmann et al }{ }^{8} \\
\text { The American Journal } \\
\text { of Surgical. } 2012\end{array}$ & 124 & $\begin{array}{l}\text { Quirodáctilos } \\
\text { Palma } \\
\text { Dorso da mão } \\
\text { Pé } \\
\text { Outros }\end{array}$ & $\begin{array}{l}60 \\
3 \\
1 \\
56 \\
4\end{array}$ \\
\hline
\end{tabular}

composto por células tumorais fusiformes ou estelares tipo fibroblastos num grau variável de matrizes mixoide ou de colágeno misturadas. À medida que o tumor persiste, a matriz colágena tende a se tornar predominante sobre a matriz mixoide. ${ }^{1}$ A neoplasia frequentemente apresenta microvascularização marcada e mastócitos. Em quase todos os casos, as figuras mitóticas são pouco frequentes, e atipias nucleares, se presentes, são de leves a moderadas. ${ }^{10}$ 


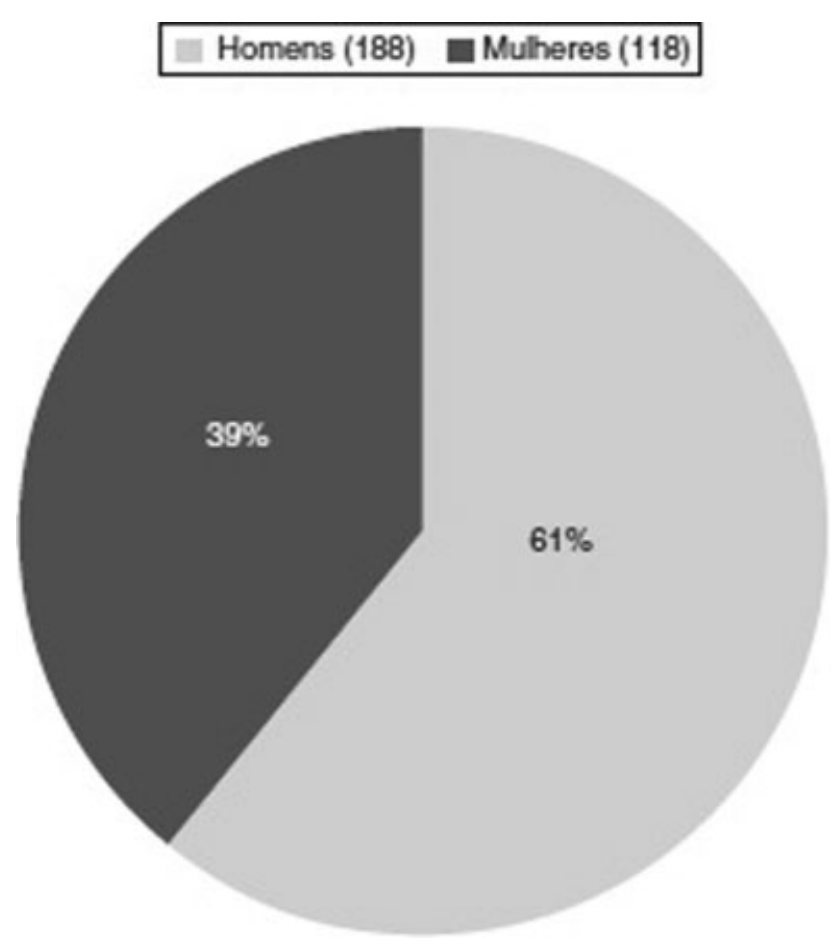

Fig. 1 Relação percentual do gênero dos casos publicados: homens, 188 casos (61\%); mulheres, 118 casos (39\%).

\section{Local de acometimento}

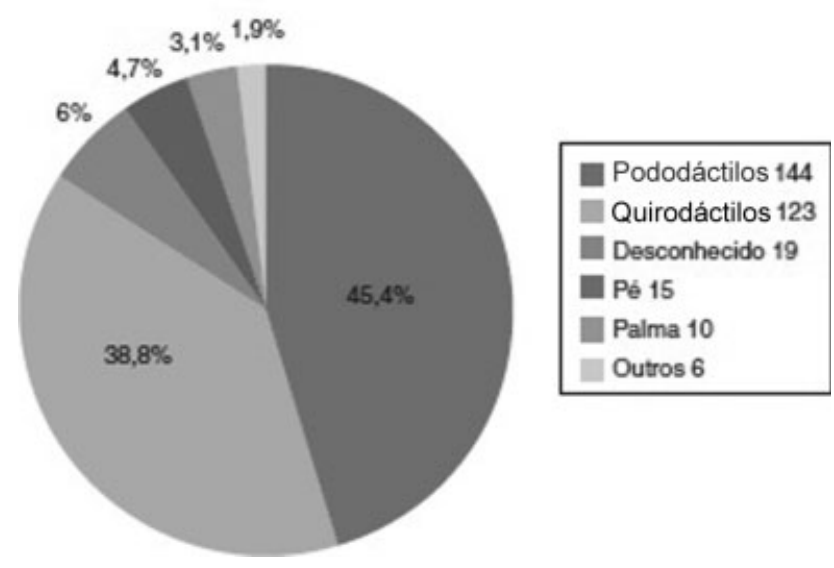

Fig. 2 Local de acometimento dos 314 casos relatados: pododáctilos, 144 casos (45,4\%); quirodáctilos, 123 casos (38,8\%); desconhecido, 19 casos (6\%); pé, 15 casos (4,7\%); palma, 10 casos (3,1\%); outros, 6 casos $(1,9 \%)$.

Imuno-histoquimicamente, as células tumorais no FAS mostram imunorreatividade para CD34, AME e CD99. No estudo de Fetsch et al, ${ }^{1}$ a positividade foi de: $21 / 23(91,3 \%)$ para CD34; 18/25 (72\%) para AME; e 11/13 (84,6\%) para CD99. Em outro estudo, de autoria de Hollmann et $\mathrm{al}^{8}{ }^{8}$ a positividade foi de apenas $42 / 61$ (68,8\%) para CD34, e $3 / 40$ (7,5\%) para AME. Não se observa positividade para actinas, desmina ou citoqueratinas. ${ }^{11}$

Os principais diagnósticos diferenciais encontrados na literatura estão representados na $\boldsymbol{- T a b e l a ~} 2$.

0 tratamento desses tumores tipicamente envolve a excisão completa para excluir a malignidade e prevenir a recorrência, segundo dez trabalhos. ${ }^{1,4,6,8,12,22,26,28,30,35}$
Tabela 2 Principais diagnósticos diferenciais e sua positividade para o CD34

\begin{tabular}{|l|l|l|}
\hline Neoplasias CD34+ & $\begin{array}{l}\text { Neoplasias } \\
\text { CD 34- }\end{array}$ & $\begin{array}{l}\text { Outras } \\
\text { lesões }\end{array}$ \\
\hline $\begin{array}{l}\text { Neurofibroma } \\
\text { mixoide }\end{array}$ & $\begin{array}{l}\text { Tumor de células } \\
\text { gigantes da } \\
\text { bainha do tendão }\end{array}$ & $\begin{array}{l}\text { Fibroma da } \\
\text { bainha do } \\
\text { tendão }\end{array}$ \\
\hline $\begin{array}{l}\text { Angiomixoma } \\
\text { superficial }\end{array}$ & Tumor glômico & Onicocriptose \\
\hline $\begin{array}{l}\text { Dermatofibrossarcoma } \\
\text { protuberans }\end{array}$ & $\begin{array}{l}\text { Perineuroma } \\
\text { esclerosante }\end{array}$ & $\begin{array}{l}\text { Mixoma } \\
\text { cutâneo }\end{array}$ \\
\hline Fibroma esclerosante & $\begin{array}{l}\text { Histiocitoma } \\
\text { fibroso benigno }\end{array}$ & \\
\hline $\begin{array}{l}\text { Mixoinflamatório acral } \\
\text { Lipoma de células } \\
\text { fusiformes }\end{array}$ & $\begin{array}{l}\text { Fibroqueratoma } \\
\text { acral }\end{array}$ & \\
\hline
\end{tabular}

\section{Discussão}

O FAS é um tumor de tecido benigno, distintivo, macio, e de crescimento lento, com predileção para a região periungueal e subungueal das mãos e dos pés. ${ }^{39}$

O FAS é uma entidade de difícil diagnóstico, devido às características que ele tem em comum com muitas patologias, o que gera atraso no diagnóstico definitivo, com casos relatados em que o intervalo entre o início da sintomatologia e o diagnóstico final foi superior a dez anos. ${ }^{9,10} \mathrm{O}$ diagnóstico tardio também se deve à sua natureza indolente e geralmente com baixa morbidade para o paciente. $O$ fato de se tratar de uma patologia relativamente recém-descrita (2001) e com poucos casos na literatura contribui para o atraso no diagnóstico e para a possibilidade de haver casos subdiagnosticados.

A divisão quanto ao gênero dos pacientes demonstra predominância para o sexo masculino de aproximadamente 2:1 em grande parte dos trabalhos avaliados ${ }^{40}$ na revisão feita no presente artigo, a predominância foi de 1,59:1: foram encontrados $61 \%$ de acometimento para o sexo masculino, enquanto o sexo feminino foi responsável por 39\%, numa população de 306 casos na qual a informação do gênero se encontrava disponível (-Fig. 1).

Até o momento não foram relatados outros fatores de risco. ${ }^{39}$

A distribuição em relação à localização demonstra a predominância das regiões acrais das mãos e dos pés, mas o FAS pode ser encontrado em outros locais, como pernas, coxas e tornozelos, conforme descrito em três trabalhos. ${ }^{7,8,25}$

Devido à heterogeneidade de apresentação e de características clínicas e histológicas, o FAS deve sempre fazer parte do diagnóstico diferencial diante de uma lesão na região acral das mãos e dos pés.

O diagnóstico é de suspeita clínica e confirmação histopatológica; porém, outros métodos diagnósticos têm sido descritos de forma auxiliar, como radiografia simples, ultrassonografia, e ressonância nuclear magnética (RNM). ${ }^{8,41,42}$

A radiografia simples do local tem sido útil na determinação do acometimento ósseo (36\% dos casos no estudo de Hollmann et $\mathrm{al}^{8}$ apresentaram lesões ósseas erosivas ou líticas). ${ }^{8}$ A massa de tecido mole normalmente não demonstra calcificação em 
radiografias simples. No entanto, é responsável pela erosão do osso cortical subjacente, que pode estar presente. Na suspeita diagnóstica, é imperativa a complementação com radiografias simples, visto que o acometimento ósseo pode alterar a extensão estimada da lesão, bem como o planejamento terapêutico, como observado no estudo de Varikatt et al. ${ }^{18}$

O exame ultrassonográfico pode acrescentar informações importantes quanto ao tamanho da massa tumoral, à localização, à forma e ao conteúdo da massa. Associado à complementação por meio do estudo vascular proporcionado pelo Doppler colorido, o padrão vascular pode ser definido, auxiliando na decisão terapêutica. 0 exame ultrassonográfico tem seu uso associado também na monitoração e recidiva das lesões tratadas cirurgicamente. ${ }^{41}$

No exame de ressonância magnética, o FAS classicamente demonstra hiperintensidade homogênea em imagens ponderadas em T2, e aumento do contraste com o tecido normal na ressonância magnética pós-contraste. ${ }^{42}$

Histopatologicamente, o FAS é descrito na maioria dos estudos como um tumor dérmico bem circunscrito, não encapsulado, composto de células fusiformes e estreladas em um arranjo fascicular solto, embutido em um estroma mixoide, mixocolagenoso ou somente de colágeno. Geralmente, ocupa toda a derme, e pode estender-se para o tecido subcutâneo ou, ocasionalmente, para a fáscia e os ossos. ${ }^{10}$

As células tumorais no FAS mostram imunorreatividade para CD34, AME e CD99. ${ }^{1}$ Embora a imunorreatividade seja difusa e relativamente forte para CD34 e CD99, para AME ela é um pouco mais variável. Recentemente, alguns tumores também mostraram reatividade focal para actina e desmina de músculo liso, embora essa não seja considerada uma característica geral. ${ }^{1,8,43}$

O diagnóstico diferencial inclui uma variedade de proliferações fibromixoides ou mixoides. O dermatofibrossarcoma protuberans (DFSP) pode ter áreas mixoides extensas que podem imitar o FAS. Esse fato, em combinação com a expressão de CD34 e AME por ambas as entidades, torna um desafio diagnóstico diferencial. ${ }^{44}$

Outros sarcomas mixoides, como o mixofibrossarcoma, podem ser distinguidos pela presença de maior atipia citológica, maior número de células em mitose, e formas mitóticas atípicas. $^{8}$

Neurofribroma mixoide geralmente tem uma aparência neural, sem aumento da vascularização. Os tumores são caracterizados por células tumorais positivas para S100 misturadas com células fibroblásticas positivas para CD34, o que difere dos FASs, os quais são negativos para S100 e têm microvasculatura concentrada. ${ }^{10,11}$

O fibroma da bainha do tendão é um tumor solitário, de crescimento lento, subcutâneo, com uma predileção para os dedos, mãos e pulsos de adultos de meia-idade. É mais comum em homens. De modo geral, os tumores são cinzento-esbranquiçados, bem circunscritos, e anexam uma bainha do tendão. Em contraste com uma proliferação celular de células fusiformes e estreladas semelhantes a fibroblastos observadas no FAS, esse tumor apresenta células estelares esparsas encaixadas numa matriz fibrocolagenosa com canais vasculares dilatados ou semelhantes a fendas. ${ }^{45}$
O tumor de células gigantes da bainha do tendão é um tumor benigno de crescimento lento, com uma predileção pela superfície dorsal do dedo perto da articulação interfalangeana distal em adultos jovens. Os tumores são lobulados, cinzento-amarronzados, e acoplados geralmente a uma bainha do tendão. Histologicamente, são fusiformes, e são misturados em um estroma de colágeno. As células gigantes multinucleadas são características. 0 pigmento de hemossiderina, as células de xantoma e as células inflamatórias crônicas também são um achado comum. As células mononucleares mostram imunorreatividade para CD68, mas não para AME, CD34 ou actina de músculo liso. ${ }^{45}$

Tumor glômico é uma massa extremamente dolorosa, solitária, subcutânea, que envolve os dedos das mãos e dos pés em adultos de meia-idade. A região subungueal pode ser afetada. As células tumorais mostram imunorreatividade para vimentina e actina de músculo liso no citoplasma. ${ }^{45}$

Em resumo, o FAS deve ser incluído no diagnóstico diferencial de qualquer massa ou nódulo de crescimento lento localizado na região periungueal ou subungueal dos dedos das mãos e dos pés, como recomendado pelos estudos avaliados nesta revisão. $4,8,10,11,22,34,35,42$

O tratamento desses tumores envolve a exérese para excluir a malignidade e prevenir a recorrência. Embora o tumor não apresente evidência de comportamento agressivo, a presença de atipia citológica em um pequeno número de casos torna o potencial de transformação maligna pouco claro. ${ }^{1}$

Não foram relatados casos de malignização na literatura. ${ }^{10}$

O acompanhamento regular após a exérese é justificado, uma vez que a taxa de recorrência relatada pode chegar a $24 \%$, principalmente devido à exérese incompleta. ${ }^{5-8}$

\section{Conclusão}

O FAS é um tumor de tecido mole único, com uma predileção para as regiões periungueais e subungueais acrais. Apresenta quadro clínico e características histopatológicas semelhantes a outras condições dermatológicas, o que dificulta o seu diagnóstico final.

Os métodos descritos na literatura que auxiliam a definição diagnóstica são: os exames de imagem, a avaliação histopatológica, e o estudo imuno-histoquímico.

Atualmente, o tratamento do FAS é limitado à exérese simples, com taxa de recidiva importante e malignização desconhecida.

Conflitos de Interesse

Os autores declaram não haver conflitos de interesse.

\section{Referências}

1 Fetsch JF, Laskin WB, Miettinen M. Superficial acral fibromyxoma: a clinicopathologic and immunohistochemical analysis of 37 cases of a distinctive soft tissue tumor with a predilection for the fingers and toes. Hum Pathol 2001;32(07):704-714

2 Ashby-Richardson H, Rogers GS, Stadecker MJ. Superficial acral fibromyxoma: an overview. Arch Pathol Lab Med 2011;135(08): 1064-1066 
3 Wakabayashi Y, Nakai N, Takenaka H, Katoh N. Superficial acral fibromyxoma of the great toe: case report and mini-review of the literature. Acta Dermatovenerol Croat 2012;20(04):263-266

4 Messeguer F, Nagore E, Agustí-Mejias A, Traves V. Fibromixoma acral superficial, un tumor periungueal CD34 positivo. Actas Dermosifiliogr 2012;103(01):67-69

5 Wei C, Fleegler EJ. Superficial acral fibromyxoma of the thumb. Eplasty 2013;13:ic13

6 Prescott RJ, Husain EA, Abdellaoui A, et al. Superficial acral fibromyxoma: a clinicopathological study of new 41 cases from the U.K.: should myxoma (NOS) and fibroma (NOS) continue as part of 21st-century reporting? Br J Dermatol 2008;159(06): 1315-1321

7 Al-Daraji WI, Miettinen M. Superficial acral fibromyxoma: a clinicopathological analysis of 32 tumors including 4 in the heel. J Cutan Pathol 2008;35(11):1020-1026

8 Hollmann TJ, Bovée JV, Fletcher CD. Digital fibromyxoma (superficial acral fibromyxoma): a detailed characterization of 124 cases. Am J Surg Pathol 2012;36(06):789-798

9 Quaba O, Evans A, Al-Nafussi AA, Nassan A. Superficial acral fibromyxoma. Br J Plast Surg 2005;58(04):561-564

10 André J, Theunis A, Richert B, de Saint-Aubain N. Superficial acral fibromyxoma: clinical and pathological features. Am J Dermatopathol 2004;26(06):472-474

11 Oteo-Alvaro A, Meizoso T, Scarpellini A, Ballestín C, Pérez-Espejo G. Superficial acral fibromyxoma of the toe, with erosion of the distal phalanx. A clinical report. Arch Orthop Trauma Surg 2008; 128(03):271-274

12 Abou-Nukta F, Fiedler P, Parkash V, Arons J. Superficial acral fibromyxoma of the distal phalanx of the thumb. J Hand Surg [Br] 2006;31(06):619-620

13 Meyerle JH, Keller RA, Krivda SJ. Superficial acral fibromyxoma of the index finger. J Am Acad Dermatol 2004;50(01):134-136

14 Kazakov DV, Mentzel T, Burg G, Kempf W. Superficial acral fibromyxoma: report of two cases. Dermatology 2002;205(03):285-288

15 Tardío JC, Butrón M, Martín-Fragueiro LM. Superficial acral fibromyxoma: report of 4 cases with CD10 expression and lipomatous component, two previously underrecognized features. Am J Dermatopathol 2008;30(05):431-435

16 Pasquinelli G, Foroni L, Papadopoulos F, Dicandia L, Bisceglia M. Superficial acral fibromyxoma: immunohistochemical and ultrastructural analysis of a case, with literature review. Ultrastruct Pathol 2009;33(06):293-301

17 Misago N, Ohkawa T, Yanai T, Narisawa Y. Superficial acral fibromyxoma on the tip of the big toe: expression of CD10 and nestin. J Eur Acad Dermatol Venereol 2008;22(02):255-257

18 Varikatt W, Soper J, Simmons G, Dave C, Munk J, Bonar F. Superficial acral fibromyxoma: a report of two cases with radiological findings. Skeletal Radiol 2008;37(06):499-503

19 Wang QF, Pu Y, Wu YY, Wang J. [Superficial acral fibromyxoma of finger: report of a case with review of literature]. Zhonghua Bing Li Xue Za Zhi 2009;38(10):682-685

20 Luzar B, Calonje E. Superficial acral fibromyxoma: clinicopathological study of 14 cases with emphasis on a cellular variant. Histopathology 2009;54(03):375-377

21 Chattopadhyay M, Farrant P, Higgins E, Hay R, Calonje E. A nodular lesion of the toe. Superficial acral fibromyxoma (SAF). Clin Exp Dermatol 2010;35(07):807-809

22 Goo J, Jung YJ, Kim JH, Lee SY, Ahn SK. A case of recurrent superficial acral fibromyxoma. Ann Dermatol 2010;22(01):110-113

23 Fanti PA, Dika E, Piraccini BM, Infusino SD, Baraldi C, Misciali C. Superficial acral fibromyxoma: a clinicopathological and immunohistochemical analysis of 12 cases of a distinctive soft tissue tumor with a predilection for the fingers and toes. $G$ Ital Dermatol Venereol 2011;146(04):283-287

24 Park SW, Kim JH, Shin HT, et al. Superficial acral fibromyxoma on the palm. Ann Dermatol 2014;26(01):123-124

25 Agaimy A, Michal M, Giedl J, Hadravsky L, Michal M. Superficial acral fibromyxoma: clinicopathological, immunohistochemical, and molecular study of 11 cases highlighting frequent Rb1 loss/ deletions. Hum Pathol 2017;60:192-198

26 Grigore LE, Baican CI, Botar-Jid C, et al. Clinico-pathologic, dermoscopic and ultrasound examination of a rare acral tumour involving the nail - case report and review of the literature. Clujul Med 2016;89(01):160-164

27 Carranza C, Molina-Ruiz AM, Pérez de la Fuente T, Kutzner H, Requena L, Santonja C. Subungual acral fibromyxomainvolving the bone: a mimicker of malignancy. Am J Dermatopathol 2015; 37(07):555-559

28 DeFroda SF, Starr A, Katarincic JA. Superficial acral fibromyxoma: A case report. J Orthop 2016;14(01):23-25

29 Cogrel O, Stanislas S, Coindre JM, et al. [Superficial acral fibromyxoma: three cases]. Ann Dermatol Venereol 2010;137(12):789-793

30 Sundaramurthy N, Parthasarathy J, Mahipathy SR, Durairaj AR. Superficial acral fibromyxoma: a rare entity - A case report. J Clin Diagn Res 2016;10(09):PD03-PD05

31 Ramya C, Nayak C, Tambe S. Superficial acral fibromyxoma. Indian J Dermatol 2016;61(04):457-459

32 Raghupathi DS, Krishnamurthy J, Kakoti LM. Cytological diagnosis of superficial acral fibromyxoma: A case report. J Cytol 2015;32 (01):39-41

33 Chabbab F, Metz T, Saez Beltran L, Theunis A, Richert B. [Superficial acral fibromyxoma in a sub-matricial location: An unusual variant]. Ann Dermatol Venereol 2014;141(02):94-105

34 Schwager ZA, Mannava KA, Mannava S, Telang GH, RobinsonBostom L, Jellinek NJ. Superficial acral fibromyxoma and other slow-growing tumors in acral areas. Cutis 2015;95(02):E15-E19

35 García AM, Mendonça FM, Cejudo MP, Martínez FM, Martín JJ. Superficial Acral Fibromyxoma involving the nail's apparatus. Case report and literature review. An Bras Dermatol 2014;89 (01):147-149

36 Hwang SM, Cho KH, Lim KR, Jung YH, Kim Song J. Superficial acral fibromyxoma on the second toe. Arch Plast Surg 2013;40(04): 477-479

37 Kwok C, Merchant WJ, Hussain W. Superficial acral fibromyxoma presenting as a haemorrhagic pigmented streak on the toenail. Clin Exp Dermatol 2013;38(05):553-554

38 Ben Brahim E, Bouabdellah M, Khanchel F, Jouini R, Abdelmoula S, Chadli-Debbiche A. Superficial acral fibromyxoma. Tunis Med 2012;90(04):340-341

39 Sawaya JL, Khachemoune A. Superficial acral fibromyxoma. Int J Dermatol 2015;54(05):499-508

40 Ashby-Richardson H, Rogers GS, Stadecker MJ. Superficial acral fibromyxoma: an overview. Arch Pathol Lab Med 2011;135(08):1064-1066

41 Baek HJ, Lee SJ, Cho KH, et al. Subungual tumors: clinicopathologic correlation with US and MR imaging findings. Radiographics 2010;30(06):1621-1636

42 Bindra J, Doherty M, Hunter JC. Superficial acral fibromyxoma. Radiol Case Rep 2015;7(03):751

43 Durda J, Kazlouskaya V, Blochin E, Maguire CA, Elston D. Dermpath quiz. Indian Dermatol Online J 2013;4(03):244-245

44 Mentzel T, Schärer L, Kazakov DV, Michal M. Myxoid dermatofibrosarcoma protuberans: clinicopathologic, immunohistochemical, and molecular analysis of eight cases. Am J Dermatopathol 2007;29 (05):443-448

45 Weedon D. Skin pathology. 2nd ed. London: Elsevier Science Ltd; 2002 